\title{
Laryngeal Involvement in Systemic Lupus Erythematosus
}

\author{
Soukaina Oumlil ${ }^{1}$, Jaouad Yousfi ${ }^{1 *}$, Mouna Zahlane ${ }^{1}$, Lamiaa Essaadouni ${ }^{1}$
}

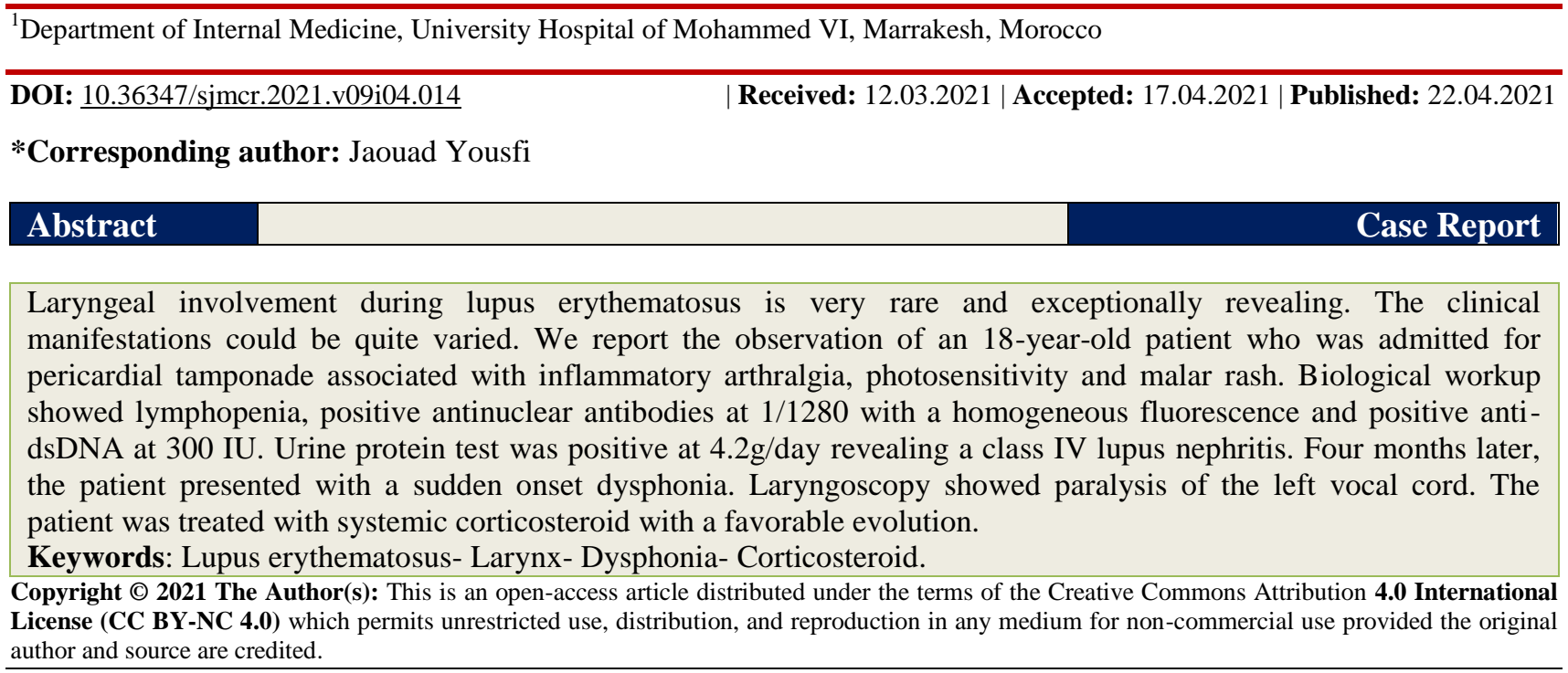

\section{INTRODUCTION}

Laryngeal involvement in systemic lupus erythematosus (SLE), first described by Scarpelli DG [1] in 1959, is rare and often asymptomatic. It occurs mostly during SLE flares, with a usually favorable outcome, and is only exceptionally severe with lifethreatening laryngeal obstruction.

\section{OBSERVATION}

A18-year-old female patient was diagnosed with SLE revealed by pericardial tamponade. In addition, the patient presented with bilateral inflammatory arthralgia, malar rash and photosensitivity. Initial workup revealed lymphopenia and a biological inflammatory syndrome. Antinuclear antibodies were positive at $1 / 1280$ with a homogeneous fluorescence. anti-dsDNA and anti-Sm antibodies were positive at $320 \mathrm{IU}$ and $45 \mathrm{IU}$ respectively. Urine protein test was positive at $4.2 \mathrm{~g} / 24 \mathrm{~h}$. Renal biopsy was in favor of lupus nephritis class IV.

The patient was treated with systemic corticosteroids, synthetic antimalarial drugs and monthly cyclophosphamide for both renal and cardiac involvement.

Four months later, the patient presented with a sudden onset dysphonia without dyspnea or dysphagia. Cervical CT- scan revealed no abnormalities, while laryngoscopy revealed left vocal paralysis without mucosal thickening or inflammatory manifestations. The diagnosis of laryngeal involvement with SLE was thus made. High-dose corticosteroid therapy was resumed at $1 \mathrm{mg} / \mathrm{kg} /$ day. The evolution was favorable with significant improvement in dysphonia and no subsequent complications.

\section{DISCUSSION}

SLE is an autoimmune disease characterized by skin, joint, hematological and visceral involvement. Otorhinolaryngologic (ENT) manifestations during SLE are very rare, and include cervical polyadenopathies, laryngeal involvement, facial paralysis, trigeminal neuralgia and other less frequent and non-specific abnormalities [2].

Laryngeal involvement is rare in SLE. It usually occurs during a flare with visceral involvement. But cases of inactive lupus with ENT involvement have been reported.

This could be due to the immune complex deposition in the laryngeal mucosa or muscle(s) or recurrent laryngeal neuropathy revealed by either a vasculitis involving vasa nervorum, neuritis or dilated pulmonary artery [3, 4]. In our case, vocal cord paralysis is thought to be caused by recurrent laryngeal neuropathy due to vasa nervorum involvement. 
The clinical manifestations are quite varied, with the main signs being dysphonia, dyspnea, pain on swallowing or speaking. However, most cases are asymptomatic. Tsunoda et al., [5] reported one case where SLE was revealed with dysphonia, while Korbet et al., [6] described a case of dysphonia, foreign body sensation and respiratory difficulties that occurred during SLE. Martin et al., [7] performed tracheotomy in 2 of their 4 patients with acute respiratory distress syndrome. Burgess and Render [8] reported a case in which the patient had a hoarse voice and dysphagia due to SLE-associated laryngopharyngitis.

Clinical examination usually reveals inflammation with occasional necrosis, laryngeal paralysis, cricoarytenoid arthritis [9, 10], mild ulceration of the laryngeal mucosa, laryngeal edema involving the vocal cords and the arytenoid mucosa. In a series of 97 cases, vocal cord paralysis and laryngeal edema were the two main clinical manifestations of this entity ( $28 \%$ and $11 \%$ of cases) [11].

Our patient presented with an isolated dysphonia. The endoscopic examination had shown paralysis of the left vocal cord without mucosal thickening.

Systemic glucocorticoids have been the mainstay of treatment in SLE with laryngeal involvement, however some cases went on to develop obstructive respiratory distress warranting emergency interventions such as tracheotomy and intubation [12].

\section{Conclusion}

Although laryngeal involvement is rare, foreign body sensation, dysphonia, or respiratory distress in patients with SLE should be suggestive of laryngeal involvement. Similarly, SLE should be considered in any young woman presenting with laryngeal involvement with inconclusive investigation results.

\section{REFERENCES}

1. Scarpelli DG, McCOY FW, Scott JK. Acute lupus erythematosus with laryngeal involvement. N Engl J Med. 1 Oct 1959;261:691-4.
2. Papadimitraki ED, Kyrmizakis DE, Kritikos I, Boumpas DT. Ear-nose-throat manifestations of autoimmune rheumatic diseases. Clin Exp Rheumatol. Août 2004;22(4):485-94.

3. Asherson RA, Hughes GR. Vocal cord paralysis in systemic lupus erythematosus complicated by pulmonary hypertension. J Rheumatol. Oct 1985;12(5):1029-30.

4. Toubi E, Naschitz JE, Kessel A, Fradis M. Vocal cord paralysis in a patient with active systemic lupus erythematosus. Isr Med Assoc J IMAJ. Mars 2000;2(3):243-4.

5. Tsunoda K, Soda Y. Hoarseness as the initial manifestation of systemic lupus erythematosus. J Laryngol Amp Otol. 1996;110(5):478-9.

6. Korbet SM, Block LJ, Lewis EJ. Laryngeal complications in a patient with inactive systemic lupus erythematosus. Arch Intern Med. Sept 1984;144(9):1867-8.

7. Martin L, Edworthy SM, Ryan JP, Fritzler MJ. Upper airway disease in systemic lupus erythematosus: a report of 4 cases and a review of the literature. J Rheumatol. Août 1992;19(8):1186-90.

8. Burgess ED, Render KC. Hypopharyngeal obstruction in lupus erythematosus. Ann Intern Med. Févr 1984;100(2):319.

9. Dufour X. Pathologie pharyngolaryngée et médecine interne. Rev Médecine Interne. 2011;1789(1001):S1-200.

10. Nanke Y, Kotake S, Yonemoto K, Hara M, Hasegawa M, Kamatani N. Cricoarytenoid arthritis with rheumatoid arthritis and systemic lupus erythematosus. J Rheumatol. Mars 2001;28(3):624-6.

11. Teitel AD, MacKenzie CR, Stern R, Paget SA. Laryngeal involvement in systemic lupus erythematosus. Semin Arthritis Rheum. Déc 1992;22(3):203-14.

12. Karim A, Ahmed S, Siddiqui R, Marder GS, Mattana J. Severe upper airway obstruction from cricoarytenoiditis as the sole presenting manifestation of a systemic lupus erythematosus flare. Chest. mars 2002;121(3):990-3. 